\title{
Emergency Surgical Management of Lumbar Burst Fracture in a Patient with COVID-19: A Case Report
}

\author{
Kurenai Hachiya $^{1)}$, Soya Kawabata ${ }^{1)}$, Sota Nagai ${ }^{1)}$, Hiroki Takeda ${ }^{2)}$, Daiki Ikeda ${ }^{1)}$, Mitsunaga Iwata ${ }^{3)}$, Shinjiro Kaneko ${ }^{2)}$ and \\ Nobuyuki Fujita ${ }^{1)}$ \\ 1) Department of Orthopaedic Surgery, School of Medicine, Fujita Health University, Aichi, Japan \\ 2) Department of Spine and Spinal Cord Surgery, School of Medicine, Fujita Health University, Aichi, Japan \\ 3) Department of Emergency and General Internal Medicine, School of Medicine, Fujita Health University, Aichi, Japan
}

\begin{abstract}
Keywords:
emergency surgery, spine trauma, burst fracture, COVID-19, pandemic
\end{abstract}

The coronavirus disease 2019 (COVID-19) pandemic is affecting healthcare systems worldwide ${ }^{1)}$. Although the number of patients with trauma has decreased during the pandemic $^{2)}$, spinal trauma patients require emergency surgery while positive for COVID-19. Here, we report the case of a patient who tested positive for COVID-19 and subsequently sustained a severe lumbar burst fracture.

A 51-year-old woman was diagnosed with moderate COVID-19 pneumonia by polymerase chain reaction testing and chest computed tomography (CT) (Fig. 1A). She was urgently admitted to the hospital for continuous follow-up, but she fell from the second floor of the hospital during the day and was transported to our hospital in an ambulance with a diagnosis of lumbar burst fracture. She complained of severe back pain; however, no paralysis of the lower limbs and no bladder and bowel dysfunction were observed. Axial view on lumbar CT revealed that $65 \%$ of the spinal canal was occupied by bone fragments at the L2 level (Fig. 1B). Lumbar magnetic resonance imaging revealed that the bone fragments severely compressed the cauda equina at the level of the fracture (Fig. 1C). We decided to conduct emergency surgery considering the high probability of development of neurological deficits due to the instability of the fracture. All staff involved in the emergency surgery, including orthopedic surgeons, anesthesiologists, nurses, and radiologists, had been fully vaccinated for COVID-19. The emergency surgery was conducted by staff with adequate personal protective equipment, including N95 masks, in an isolated negative-pressure operating room (Fig. 2A). The equipment in the operating room was covered with plastic bags as much as possible (Fig. 2A). Percutaneous pedicle screw fixation was performed at one level above and one level below the fracture level (Fig. 2B). The patient was extubated in the operation room and was then transferred to the intensive care unit. No treatment was given for pneumonia during hospitalization. The data on the arterial blood gas parameters preoperatively and at the first and second postoperative day are shown in Supplementary Table 1. Rehabilitation therapy in the bedside was started from the first postoperative day. Ambulation with a hard brace was permitted at postoperative day 6. Postoperative CT revealed that the percentage of spinal canal occupied by bone fragments had improved to $25 \%$ at the fracture level (Fig. 3A). At postoperative days 6 (Fig. 3B) and 15 (Fig. 3C), COVID-19 pneumonia was confirmed to have improved by chest CT. No staff developed COVID-19 infection symptoms after the surgery.

To the best of our knowledge, a few institutions have presented flow charts for emergency spinal surgery during the COVID-19 pandemic ${ }^{3,4}$. Our literature review retrieved two cases of emergency spinal surgery for patients who were suspected of having or positive for COVID-19. In both cases, the spread of the virus was well controlled and the patients had good treatment outcomes ${ }^{5,6)}$. In the presented case, we also successfully conducted the emergency surgery with adequate COVID-19 infection control. Because the patient had no paralysis of the lower limbs and no bladder and bowel dysfunction, elective surgery was a treatment option. However, considering that early surgical intervention can

Corresponding author: Nobuyuki Fujita, nfujita2007@gmail.com

Received: October 7, 2021, Accepted: November 12, 2021, Advance Publication: February 10, 2022

Copyright (C) 2022 The Japanese Society for Spine Surgery and Related Research 

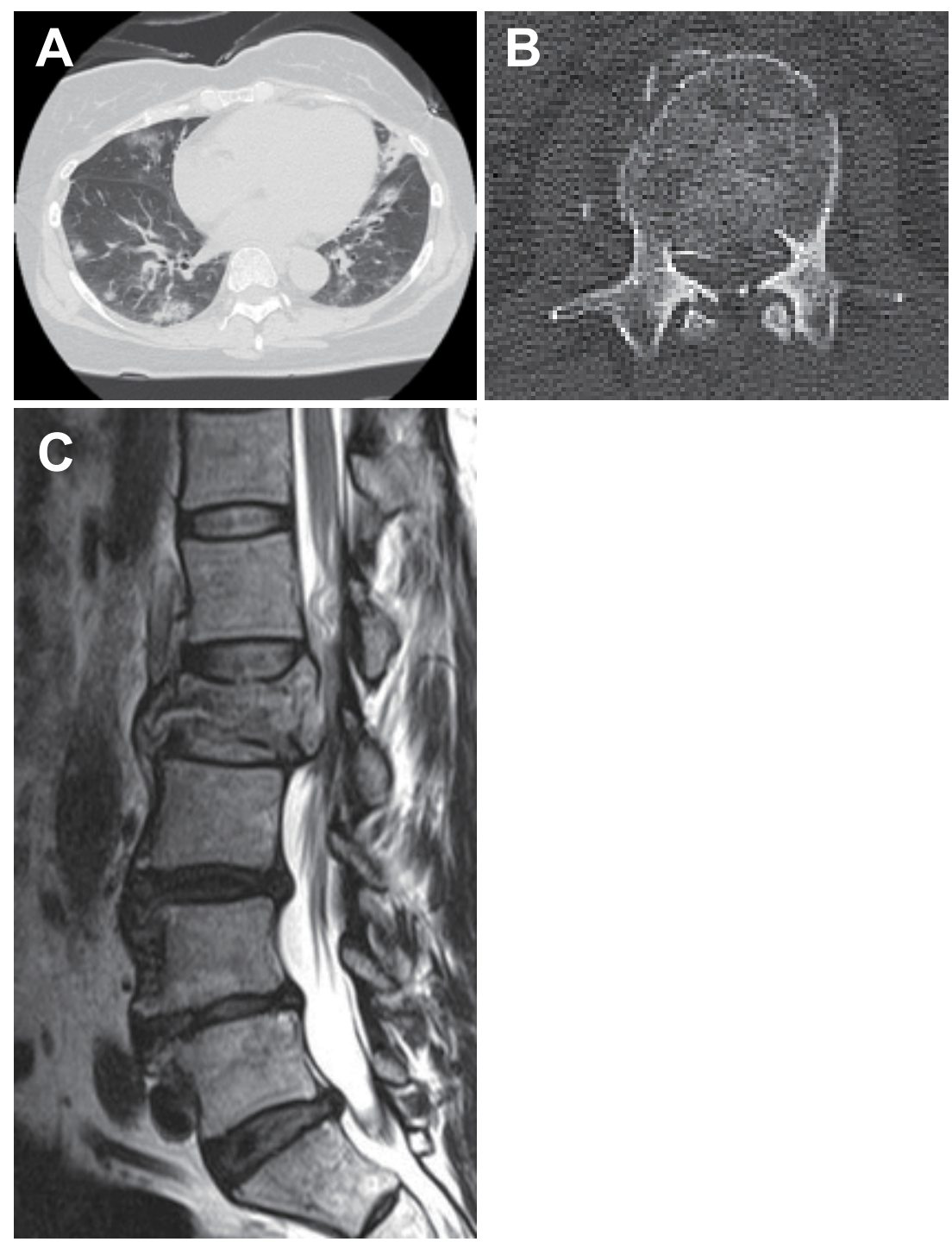

Figure 1. Preoperative condition of the COVID-19 patient with lumbar burst fracture. Chest computed tomography (CT) on axial view (A), lumbar axial CT at the L2 level (B), and T2-weighted magnetic resonance imaging on sagittal view (C).

improve pneumonia by early ambulation and facilitate reduction of the spinal fracture due to ligamentous taxis, we chose emergency spinal surgery. The COVID-19 pandemic has created the need for special organizations within medical institutions for surgical treatment of patients with COVID19. Additionally, multidisciplinary plans are required for patients with COVID-19 to protect other patients and the medical staff. Fig. 4 shows the flow-chart and the safety checklist in our institution for emergency surgery of spinal trauma patients with COVID-19. We hope that more advanced methods for prevention and treatment of COVID-19 would ease the management of spinal trauma patients with COVID-197).

Disclaimer: Nobuyuki Fujita is one of the Editors of Spine Surgery and Related Research and on the journal's Editorial Committee. He was not involved in the editorial evaluation or decision to accept this article for publication at all.
Conflicts of Interest: The authors declare that there are no relevant conflicts of interest.

Sources of Funding: There are no sources of funding that the authors acknowledge.

\section{Author Contributions:}

Conception and design: Nobuyuki Fujita.

Acquisition of data: Kurenai Hachiya, Soya Kawabata.

Interpretation of data: Sota Nagai, Hiroki Takeda, Daiki Ikeda, Mitsunaga Iwata, Shinjiro Kaneko.

Study supervision: Mitsunaga Iwata, Shinjiro Kaneko.

Drafting the manuscript: Kurenai Hachiya, Soya Kawabata, Nobuyuki Fujita.

Critically revising the manuscript: Sota Nagai, Hiroki Takeda, Daiki Ikeda, Mitsunaga Iwata, Shinjiro Kaneko.

The final version of the manuscript was approved by all authors. 
A
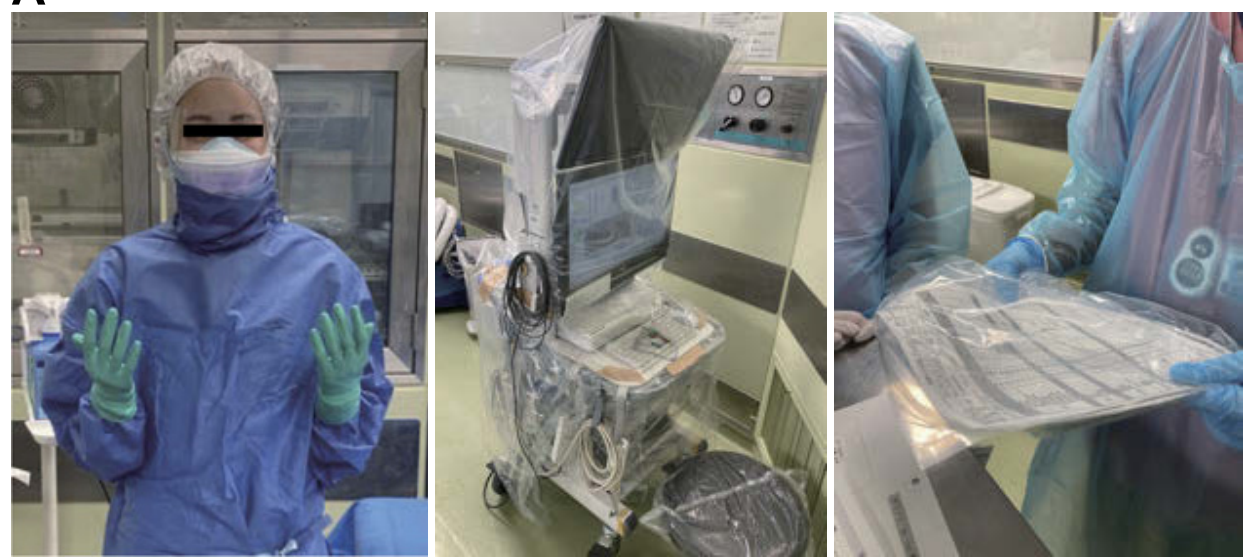

B

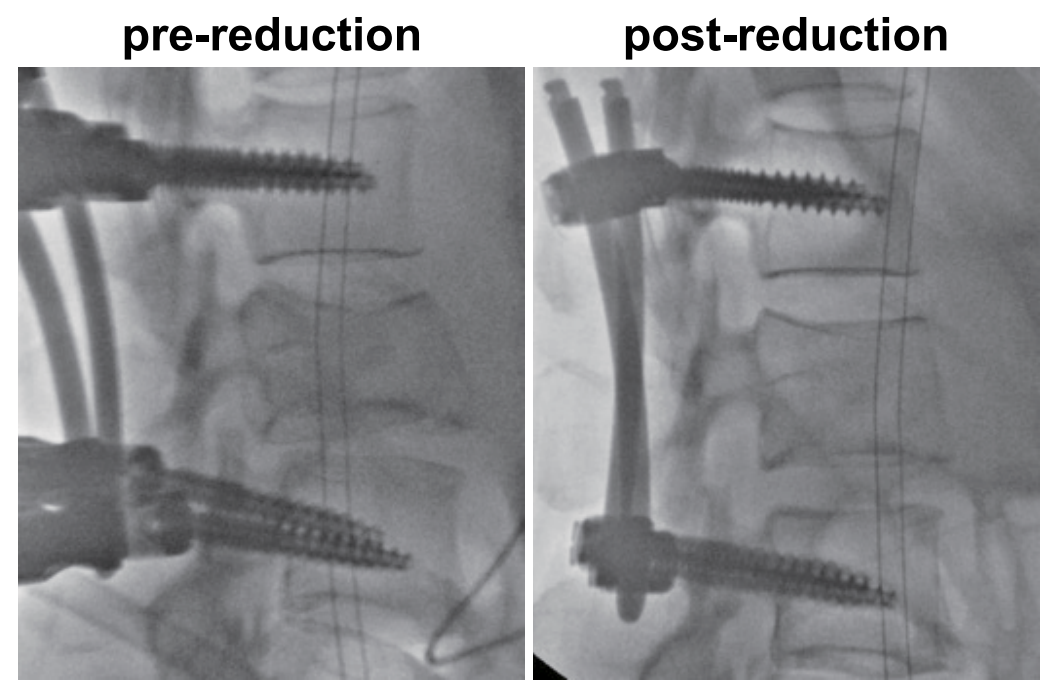

Figure 2. (A) Preparation of the operation room. (B) Intraoperative radiological findings. Lateral view of the lumbar spine at prereduction and postreduction.
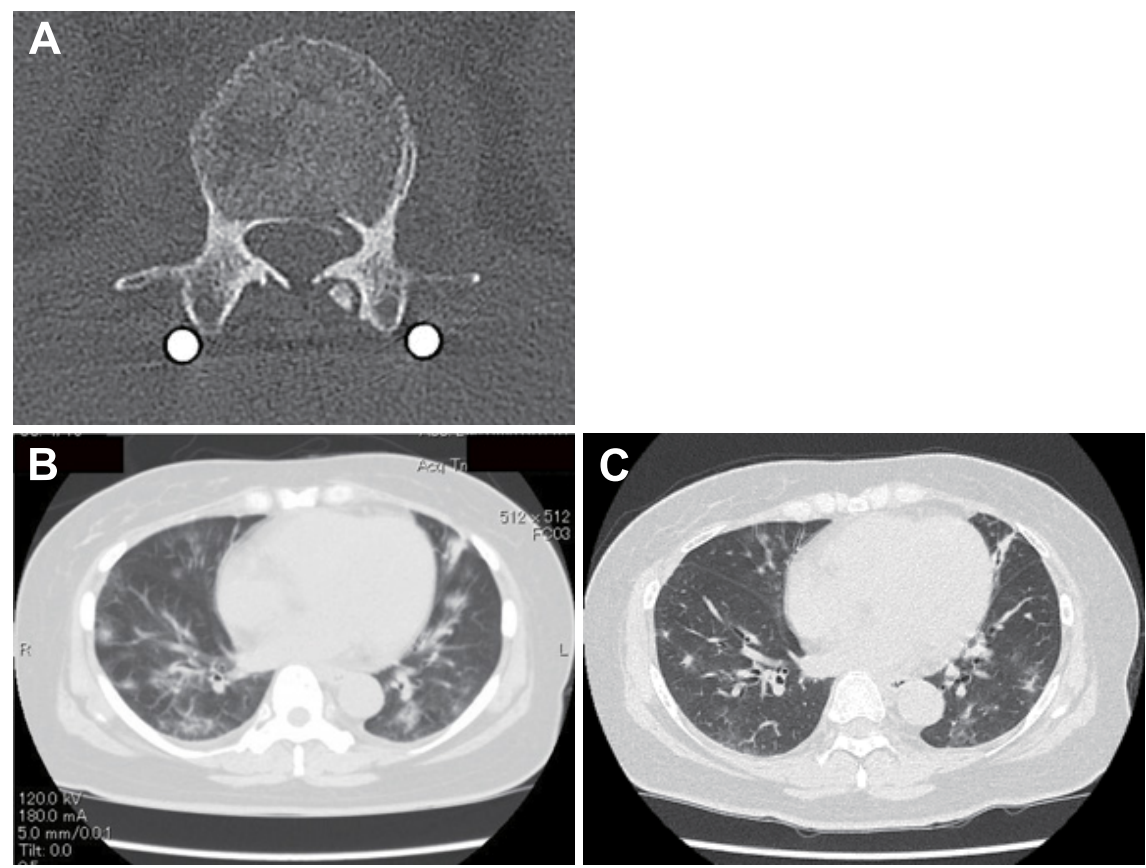

Figure 3. Postoperative condition of COVID-19 pneumonia with lumbar burst fracture. Lumbar CT at the L2 level (A) and chest CT at postoperative days 6 (B) and 15 (C). 


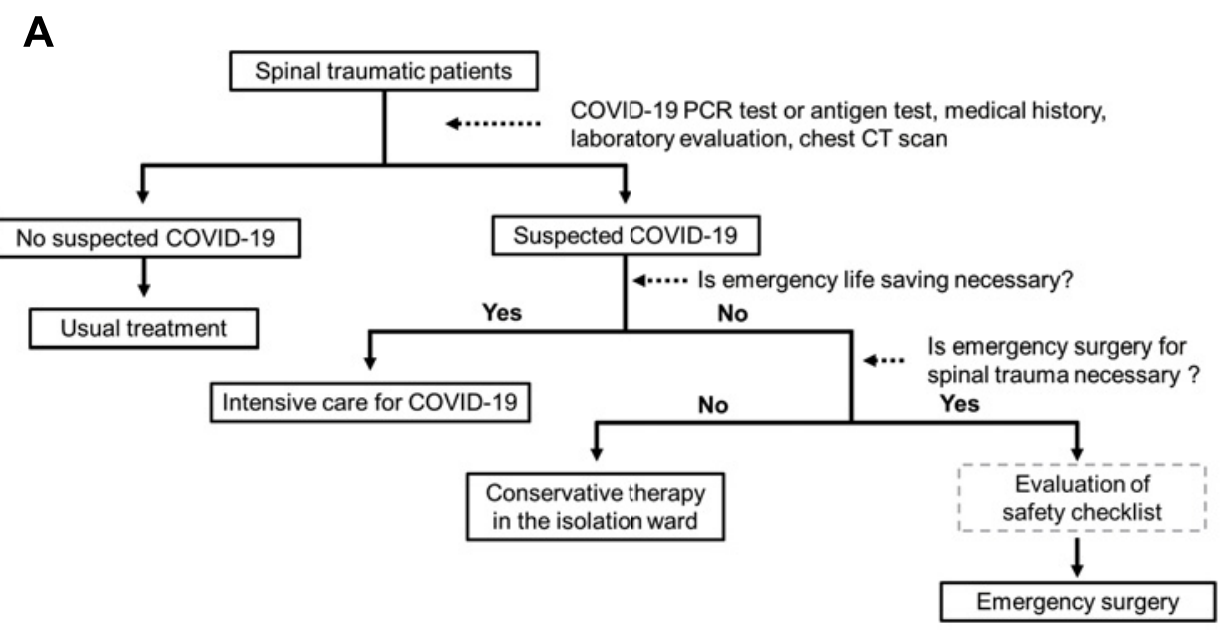

B

Safety checklist

\begin{tabular}{|c|c|}
\hline Items & This case \\
\hline $\begin{array}{c}\text { Is negative pressure } \\
\text { operation room available? }\end{array}$ \\
\hline $\begin{array}{c}\text { Have all medical staffs been fully } \\
\text { vaccinated for COVID-19? }\end{array}$ \\
\hline $\begin{array}{c}\text { Is personal protective equipment of } \\
\text { all medical staffs adequate? }\end{array}$ \\
\hline
\end{tabular}

Figure 4. Flow-chart (A) and safety checklist (B) for emergency surgery of spinal trauma patients with COVID-19.

Ethical Approval: This study was approved by the Fujita Health University Hospital Ethics Committee (approval number, HM20-530).

Informed Consent: Informed consent was obtained by the participant in this study.

\section{References}

1. Massoudi BL, Sobolevskaia D. Keep moving forward: health informatics and information management beyond the COVID-19 pandemic. Yearb Med Inform. 2021;30(1):75-83.

2. Coleman JR, Burlew CC, Platnick KB, et al. Maintaining trauma care access during the COVID-19 pandemic: an urban, level-1 trauma center's experience. Ann Surg. 2020;272(2):e58-60.

3. Wang YL, Zhu FZ, Zeng L, et al. Guideline for diagnosis and treatment of spine trauma in the epidemic of COVID-19. Chin J Traumatol. 2020;23(4):196-201.

4. Giorgi PD, Villa F, Gallazzi E, et al. The management of emer- gency spinal surgery during the COVID-19 pandemic in Italy. Bone Joint J. 2020;102-b(6):671-6.

5. Surachman AJD, Yanuarso, Akbar DL. Emergency decompression and stabilization of 1st thoracic spinal cord injury and sacral fracture in a Covid-19 patient: a case report. Int J Surg Case Rep. 2021;81:105670.

6. Alhashash M, Elsebaiy W, Farag M, et al. Emergency surgical management of cervical spine fracture-dislocation with acute paraplegia in COVID-19 (Coronavirus disease 2019)-suspected patient: first experience from a German spine centre. Eur Spine J. 2021;30 (2):468-74.

7. Rana R, Tripathi A, Kumar N, et al. A comprehensive overview on COVID-19: future perspectives. Front Cell Infect Microbiol. 2021; 11:744903.

Spine Surgery and Related Research is an Open Access journal distributed under the Creative Commons Attribution-NonCommercial-NoDerivatives 4.0 International License. To view the details of this license, please visit (https://creativeco mmons.org/licenses/by-nc-nd/4.0/). 